'Departamento de Psiquiatría, Escuela de Medicina, Pontificia Universidad Católica de Chile. Santiago de Chile.

${ }^{2}$ Clínica UC San Carlos de Apoquindo. Santiago de Chile. ${ }^{3}$ Centro de Bioética. Pontificia Universidad Católica de Chile. Santiago de Chile. ${ }^{\mathrm{a}}$ Abogado.

Fuentes de Financiamiento: Este trabajo ha sido financiado con fondos de Departamento de Psiquiatría de la Pontificia Universidad Católica de Chile.

Recibido el 27 de agosto de 2014, aceptado el 29 de enero de 2015.

Correspondencia a: Dr. Jaime Santander Toro Clínica UC San Carlos de Apoquindo.

Camino el Alba 12351,

Las Condes, Santiago. Teléfono: 27548935 jsantan@med.puc.cl jaimesantander@gmail.com

\section{Consideraciones acerca de la conducta suicida de pacientes hospitalizados y responsabilidad del médico tratante}

\author{
JAIME SANTANDER ${ }^{1,2}$, WALTER BROKERING ${ }^{2}$, \\ PAULINA RAMOS ${ }^{3, a}$, ÁNGELA ARENAS $^{\mathrm{a}}$
}

\section{Suicide in hospitalized patients and medical liability}

Suicide is a complex phenomenon that has accompanied human beings throughout history. Its strong association with mental disorders led to its medicalization and psychiatrists became the physicians in charge of diagnosing and treating patients at risk of suicide. This article discusses the potential limitations that psychiatrists may face when diagnosing suicide risk and providing optimal care. Evidence of the eventual inevitability of suicide and the tension that may arise between providing optimal treatments on the one hand and preserving the rights of patient's autonomy and dignity on the other is also presented. We propose that although diagnosing and adequately treating patients at risk of suicide would be the psychiatrist's responsibility, the act of suicide itself is personal and non-transferable. Considering the latter as part of the medical team's responsibilities would turn working with patients with mental disorders into a fearless act. Finally, suicide should be considered to be part of the natural history of the evolution of many mental disorders and, thus, should constitute a specific topic when training specialists.

(Rev Med Chile 2015; 143: 506-511)

Key words: Principle-based ethics; Psychiatry; Suicide.
$U_{y}$ 1 suicidio es un fenómeno que ha sido parte integral de la historia de la humanidad y obedece a causas complejas y variadas. Albert Camus llegó a decir que el suicidio sería el único "problema filosófico verdaderamente serio"1. A nivel mundial se estima que presenta una tasa de mortalidad anual de 14,5 por cada cien mil habitantes y en Chile esa tasa alcanza a 15,6 por 100.000 habitantes, con una lenta tendencia al alza, que se viene observando consistentemente desde 1990 a la fecha².

Una arista poco discutida y para la que existe escasa literatura es la relación que se produce justamente entre la conducta suicida y la responsabilidad médica o del equipo de salud en este tipo de situaciones, que cuando ocurren son difíciles de analizar e interpretar adecuadamente debido a la violencia y gravedad de las consecuencias de este tipo de conductas.

Se observa la necesidad de reflexionar acerca de la responsabilidad médica y de determinar la Lex Artis en estos casos. Para esto se realiza una revisión dirigida de la literatura nacional e internacional de los últimos cinco años, así como de documentos y artículos relevantes previos.

\section{La diada médico-paciente}

\section{El médico}

El suicidio es un problema humano que se ha medicalizado ${ }^{3,4}$, lo que de por sí ya genera un problema bioético ${ }^{3}$, pero que se puede entender por la alta correlación entre las conductas autolíticas $y$ enfermedad mental ${ }^{5}$. La especialidad médica 
que lidia preferentemente con este tipo de enfermedades es la psiquiatría, lo que hace que estos especialistas enfrenten la problemática del suicidio de manera más frecuente que otros médicos. El accionar del médico en general, y del psiquiatra en particular, se rige por la Lex Artis, esto es según las normas, reglas técnicas y protocolos propios de los procedimientos que se utilizan en la Ciencia Médica 6 . Por cierto que la Lex Artis, en particular la lex artis ad-hoc, está influida por pueblos, profesionales, legisladores y las circunstancias que propician un especial modo de entender y actuar las reglas, lo que genera dificultades adicionales al evaluar la responsabilidad médica ${ }^{7}$.

\section{El paciente y su cuidado}

El paciente es definido por la Organización Mundial de la Salud (OMS) como el usuario de los servicios de salud, sea por enfermedad o estando sano ${ }^{8}$. En cuanto al paciente psiquiátrico, según el ordenamiento jurídico de nuestro país, son aquellas personas que sufren de una enfermedad o trastorno mental y que se encuentren bajo supervisión o tratamiento médico especializado" (artículo 6 del Decreto Supremo N 570 del Ministerio Salud, D.O. 20 de agosto de 1998). Si bien dichos pacientes, como todas las personas en Chile, tienen derecho, cualquiera que sea el prestador que ejecute las acciones de promoción, protección y recuperación de su salud y de su rehabilitación, a que ellas sean dadas oportunamente y sin discriminación arbitraria, en las formas y condiciones que determinan la Constitución y las leyes. El legislador considera un régimen especial de atención que debe regirse por las normas que dicte el Ministerio de Salud, para asegurar que aquella sea oportuna y de igual calidad" (artículo $2^{\circ}$ Ley No 20.584, D.O. 24 de abril de 2012, sobre Derechos y Deberes que tiene las personas vinculadas a su atención en salud).

En cuanto al cuidado que se les debe otorgar a estos pacientes, el sistema nacional hace suyos los principios éticos reconocidos en diversas declaraciones de organizaciones internacionales. Al respecto, se destaca que en la Asamblea General de la Asociación Mundial de Psiquiatría en 1977 se aprobó la Declaración de Hawai ${ }^{9}$, que consagra el deber de ofrecer a estos pacientes la mejor terapéutica disponible y tratárseles con solicitud y respeto por su dignidad y autonomía. Asimismo, reconoce que la relación terapéutica entre el paciente y el psiquiatra está basada en el acuerdo, en la confianza, en la confidencialidad, en la cooperación y responsabilidad mutua, lo que distribuye equilibradamente las obligaciones, radicándolas en ambos miembros de la díada. Se reconoce también que en enfermos graves puede no ser posible establecer una relación terapéutica con las características señaladas, en cuyo caso, la declaración promueve que el psiquiatra tome contacto con familiares o personas próximas al paciente. La Declaración citada reconoce la licitud ética de administrar un tratamiento en forma compulsiva, siempre que se haga en beneficio e interés del paciente y se cuente con el consentimiento de alguien próximo al mismo.

Por su parte, la Federación Mundial para la Salud Mental adoptó, en 1989, la Declaración de Luxor $^{10}$, que se pronuncia en el mismo sentido. Finalmente, la Asamblea General de las Naciones Unidas aprobó, en 1991, un conjunto de 25 principios para la protección de los enfermos mentales y para el mejoramiento de la atención de la salud mental ${ }^{11}$. Entre los principios más importantes, destacan:

- Todas las personas que padezcan una enfermedad mental serán tratadas con humanidad y respeto a su dignidad, inherente a su condición humana.

- Se protegerá al paciente de cualquier daño, incluida la administración injustificada de medicamentos, así como de los malos tratos por parte del personal $\mathrm{u}$ otros pacientes.

- En el ámbito del tratamiento, se consagra el derecho de todo paciente a un tratamiento lo menos restrictivo posible, de acuerdo a sus necesidades de salud y de protección de terceros.

- Se exige el consentimiento del paciente para todo tratamiento, salvo en casos excepcionales, previstos.

\section{Consideraciones en relación a la conducta suicida}

\section{Conducta suicida}

El concepto de conducta suicida se refiere a un continuo que va desde ideas de suicidio hasta la consumación del suicidio, pasando por la planificación y los intentos frustros de suicidio. La OMS, como criterio operativo de suicidio ${ }^{12}$ propone:

a) que sea un acto con resultado letal;

b) deliberadamente iniciado y realizado por el sujeto; 
c) sabiendo o esperando el resultado letal y;

d) la muerte es un instrumento para obtener cambios en la actividad consciente y en el medio social.

Aunque el suicidio tiene una prevalencia mundial de 14,5 por cien mil al año, Bertolote et $\mathrm{al}^{13}$ encontraron en un estudio multinacional que el espectro suicida era mucho más frecuente, alcanzando las ideas de suicidio una prevalencia de vida de 7,3-25,4\%, la planificación de 1,1-15,6\% y los intentos de $0,4-4,2 \%$, y de estos últimos sólo habrían recibido alguna atención médica entre 22 y $88 \%$ dependiendo del país en que este se realizara.

\section{Medicalización del suicidio}

$\mathrm{Ni}$ las ideas suicidas, ni tampoco la planificación, intentos o consumación del suicidio son considerados trastornos mentales como tales, y en las clasificaciones más importantes de trastornos mentales, son considerados síntomas de constelaciones diagnósticas más amplias. Está demostrado, sin embargo, que existe una alta correlación entre el suicidio y los trastornos mentales ${ }^{14,15}$, cuyo tratamiento realizan médicos, y de aquí la progresiva medicalización del problema $^{3,4}$. Pese a esta asociación es importante no perder de vista que el suicidio es un fenómeno mucho más complejo, que trasciende los límites de la enfermedad mental y la medicina, siendo conocidos los ejemplos de conductas suicidas en otros contextos como ocurre en una serie de situaciones sociales, como manifestación de descontento social, como opción de honorabilidad, como una muerte altruista o ante enfermedades somáticas terminales o que involucran dolor o limitaciones crónicas ${ }^{16,17}$.

\section{Evaluación del riesgo suicida de un individuo}

No se dispone de ningún marcador objetivo para un observador externo de presencia de ideas suicidas ni tampoco de la inminencia de un acto con finalidad suicida. Esta es una limitación que tensiona al máximo la decisión respecto de la intensidad de las medidas de cuidado a implementar. Las posibles vías de aproximación en la evaluación del riesgo de suicidio son tres:

1) que en la entrevista médica la persona con ideas suicidas lo exprese a su tratante traspasándole a este último la responsabilidad de cuidado y ayuda, o;
2) que la conducta del sujeto sea tal que haga suponer al médico, por su experiencia y conocimiento, la inminencia de un acto suicida aunque este no haya sido explicitado o incluso se niegue, $\mathrm{o}$;

3) que el médico en conocimiento de factores de riesgo estadístico conocidos como es el sexo, la edad, variables psicosociales, tipo de trastorno mental existente, etcétera, establezca una razonable presunción de un riesgo suicida aumentado. Aunque esta hipótesis es atractiva, existe clara evidencia que muestra que la mayor parte de quienes cumplen con un alto riesgo suicida no atentarán contra su vida, en tanto un grupo significativo de suicidios consumados son realizados por personas con un perfil de riesgo suicida relativamente bajo ${ }^{18-20}$.

Sin embargo, un individuo puede no presentar ningún de los elementos descritos y aun así presentar ideación suicida. El principal problema clínico es que, a diferencia de otros padecimientos, el individuo con ideas suicidas, aun estando hospitalizado para ser tratado por algún trastorno mental, con frecuencia las niega activamente y pretende mostrar normalidad de manera de conseguir ser dado de alta para poder intentar el suicidio posteriormente ${ }^{21,22}$.

\section{Evitabilidad del suicidio}

Existen numerosos estudios referentes al suicidio de individuos bajo el cuidado de terceros, como ocurre en personas privadas de libertad en recintos penitenciarios como de enfermos bajo cuidado en hospitales generales y psiquiátricos. En Chile, entre los años 2006 y 2011, ambos incluidos, se constataron, sólo en la Región Metropolitana, 33 suicidios en centros de detención ${ }^{23}$ y 17 en recintos asistenciales $(23,5 \%$ de estos en servicios de psiquiatría $)^{24}$. Aunque evidentemente se trata de situaciones diferentes, estos hallazgos han llevado a establecer normas de alerta y cuidado cada vez más sofisticadas a fin de intentar evitar esta situación, con un éxito parcial, ya que es claro que ninguna norma ha sido capaz de evitar el suicidio, como queda demostrado por los reportes de suicidio en centros clínicos especializados ${ }^{18,21,25}$. En definitiva, aun instaurándose protocolos y normas tendientes a un cuidado intensivo y prolijo el suicidio es considerado un fenómeno inevitable ${ }^{26}$. 


\section{Autonomía del paciente}

En Chile, además de la Ley de Derechos y Deberes de los Pacientes ya mencionada, existe un reglamento que regula la internación en contra de la voluntad de un individuo ${ }^{27}$. En este reglamento se especifica que el psiquiatra puede, con ciertas restricciones, realizar este tipo de internación siempre y cuando exista riesgo suicida u homicida, o para necesidad de estudio y tratamiento bajo régimen de internación. La responsabilidad de definir la presencia de estas causales, que permiten transitoriamente posponer la autonomía de un paciente, queda entregada a la evaluación clínica del médico tratante, evaluación que tiene las limitaciones que ya hemos mencionado. La sumatoria de factores de riesgo de suicidio se asocian a la ocurrencia de actos de autoagresión durante la internación, sin embargo, la gran mayoría de los pacientes que cumplen dichos criterios no cometen dichos actos, lo que hace que la presencia de dichos factores de riesgo no baste para decidir trasponer los derechos del paciente. En todo caso, al parecer, en estudios nacionales, la internación involuntaria de urgencia (por hasta $72 \mathrm{~h}$ ) alcanza $18 \%{ }^{28}$, en tanto la internación involuntaria administrativa (por hasta 30 días) llega sólo a 3\% de los hospitalizados $^{29}$. Es evidente que en estos pacientes las normas de cuidado son más intensas, sin embargo, estas no debieran ser de esa manera en quienes están internados voluntariamente y que, por ende, se supone solicitan, o al menos aceptan, ser ayudados por su condición mental.

\section{Consideraciones acerca de la responsabilidad del psiquiatra en relación a pacientes internados en unidades psiquiátricas}

\section{Cuidado adecuado}

En el caso de pacientes internados, sería impresentable disponer para todos las mismas medidas de seguridad extremas que imposibilitarían todo acto contra su vida. Por lo demás, esta conducta implicaría lesionar los derechos y dignidad de una gran cantidad de pacientes que muy probablemente no tendrían ninguna conducta de autoagresión $^{18,19,20}$. De hecho, se considera que todo proceso de tratamiento psiquiátrico que requiera proporcionar internación a una persona con enfermedad o trastorno mental utilizará, preferentemente, el medio menos restrictivo de sus derechos y libertades personales. Corresponderá al médico tratante decidir técnicamente la elección del medio que resulte más adecuado ${ }^{27}$, y para ello requerirá realizar, en la medida de lo posible, y con las limitaciones ya comentadas, la mejor ponderación del riesgo suicida del individuo. Así, la lógica que sustenta la adopción de las medidas cautelares para contener el riesgo suicida es la misma que la que conduce al diseño del plan terapéutico, la gradualidad y proporcionalidad en el uso de los recursos. Cuanto mayor sea el riesgo y la evidencia disponible, mayor será el grado de precaución y cuidado que deberá adoptarse. Desde el punto de vista del médico, el problema para ponderar las medidas de cuidado es la dificultad en la evaluación adecuada de la suicidalidad del paciente dado la carencia de indicadores objetivos de la misma.

\section{Responsabilidad del psiquiatra}

En el caso del suicidio, determinar la lex artis ad-hoc del psiquiatra no es un tema simple. El accionar del tratante puede ser cuestionado en cuanto a una inadecuada evaluación del riesgo de suicidio o por una respuesta negligente al mismo. Incluso cuando se represente al psiquiatra una conducta omisiva, por ejemplo si no dispuso un cuidador personal y permanente para vigilar al paciente las $24 \mathrm{~h}$ de cada día, ello sólo puede haber minimizado los riesgos de suicidio, no evitarlo. Por otra parte, los constantes cambios que se producen en la evolución del paciente y por los cambios en las circunstancias particulares, hace que se hable de una "lex artis ad-hoc", que cambia en la situación que se examina, por lo que el incumplimiento de la Lex Artis general no supone una falta del cuidado debido ${ }^{30,31}$. Con todo, la omisión que algunos podrían calificar de negligente no es la causa directa y precisa de la muerte del paciente -inexistencia de nexo causal- y por ende no puede ser objeto de reproche jurídico. No es procedente exigir al psiquiatra una obligación de resultados ${ }^{32}$.

\section{Conclusiones}

El suicidio de un paciente afecta indudablemente a la familia del afectado, pero también lo hace con el equipo tratante. De hecho, entre 20\% y $51 \%$ de quienes trabajan en salud mental enfrentarían la muerte de uno o más de sus pacientes por 
esta vía ${ }^{33,34}$, con el consiguiente efecto psicológico entre estos. Aunque el suicidio es una conducta humana compleja, su frecuente asociación con patología psiquiátrica y la medicalización de este lo ha convertido en un fenómeno íntimamente asociado a la práctica médica, en particular a la práctica psiquiátrica. Se debe entender que el suicidio es uno de los resultados posibles en el diagnóstico y tratamiento de personas con trastornos mentales, y por ende se debe incluir este aspecto en la formación de los especialistas en psiquiatría ${ }^{35,36}$.

Aun con la mejor formación psiquiátrica disponible la ponderación exacta del riesgo suicida ha demostrado no ser posible, y a la fecha no existen protocolos de cuidado que eviten la concreción de actos suicidas. El diagnóstico más adecuado posible y la instauración del tratamiento y protocolos de cuidado más adecuados para cada paciente constituyen la Lex Artis esperable, empero esta continuamente debe estar en revisión, además de las dificultades ya descritas, en la evaluación de la suicidalidad. Las normas de cuidado se encuentran además en tensión permanente con la necesidad de respetar la dignidad y autonomía de los pacientes, debiendo el clínico enfrentar y estar resolviendo permanentemente estas obligaciones contradictorias. Es en esta tensión en que se juzga el cumplimiento de la Lex Artis por parte del médico.

Otro tema es la responsabilidad del acto suicida, la cual es finalmente siempre personal e intransferible. Aunque en los casos de psicosis o de alteración transitoria de la capacidad de ejercer el mejor juicio, la autoagresión puede ser considerada producto de su condición psicopatológica, en la mayoría de los casos de suicidio en hospitalizados esto ha ocurrido en individuos con circunstancias psicopatológicas que no disminuyen los derechos y autonomía del paciente, lo que ha llevado incluso a algunos autores a plantear el concepto del "suicidio racional"33 , esto es equiparar algunos casos de decisión suicida a lo observado en casos como enfermos terminales sin enfermedad psiquiátrica. Aunque estas propuestas son controversiales, ponen de manifiesto la necesidad de mantener el foco de la responsabilidad por la conducta suicida en el sujeto que la ejecuta, transferirla al médico transformaría el cuidado de personas con trastornos mentales en un acto temerario e incentivaría a que los equipos no se hagan cargo, justamente de acoger y tratar a sujetos con gran necesidad de ayuda.

\section{Referencias}

1. Camus A. El Mito de Sísifo, tercera edición. Madrid, España: Alianza Editorial; 1985.

2. Nahuelpán E, Varas J. El Suicidio en Chile: Análisis del Fenómenos desde los Datos Médicos Legales. Disponible en: http://www.sml.cl/proyectos/estadistica/documentos/SUICIDIO_EN_CHILE_2000-2008.pdf (Consultado el 2 de abril de 2014).

3. Salas AA. La Medicalización del Suicidio: un Dilema Bioético. Rev Bioética y Derecho 2011; 22: 24-35.

4. MacDonald M. The medicalization of suicide in England: laymen, physicians, and cultural change, 15001870. Milbank Q 1989; 67 Supp 1: 69-91.

5. Hawton K, Saunders K, Topiwala A, Haw C. Psychiatric disorders in patients presenting to hospital following self-harm: a systematic review. J Affect Disord 2013; 151 (3): 821-30.

6. Vallejo G. La responsabilidad penal por imprudencia del médico psiquiatra ante el suicidio del paciente con trastorno mental. Nuevo Foro Penal, [S.1.], 2013 abril; 8 (79): 56-70. ISSN 0120-8179. Disponible en: http://publicaciones.eafit.edu.co/index.php/nuevo-foro-penal/article/ view/1909/1919 [Consultado el 23 de junio de 2014].

7. Valencia G. La Lex Artis. Revista Médico Legal 2001; 7 (3): 21-25. Disponible en: http://www.medicolegal.com. co/pdf/esp/2001/7/3/asp_resp_2_v7_r3.pdf [Consultado el 23 de junio de 2014].

8. World Health Organization. A declaration on the promotion of patient's rights in Europe. Disponible en: http://www.who.int/genomics/public/eu_declaration1994.pdf?ua=1 [Consultado el 23 de junio de 2014].

9. World Psychiatric Association. Declaration of Hawaii. J Med Ethics 1978; 4 (2): 71-3.

10. World Federation of Mental Health. Declaration of Luxor: Declaration of Human Rights and Mental Health, 1989. Disponible en: http://wfmh.com/wp-content/ uploads/2013/12/DeclarationHR_MH.pdf [Consultado el 2 de abril de 2014].

11. United Nations. Principles for the Protection of Persons with Mental Illness and the Improvemente of Mental Health. Addopted by General Assembly resolution 46/119 of 17 Decembre 1991. Disponible en: http:// www.who.int/mental_health/policy/en/UN_Resolution_on_protection_of_persons_with_mental_illness. pdf [Consultado el 2 de abril de 2014].

12. Soler PA, Gascón J. RTM-III: Recomendaciones Terapéuticas en los Trastornos Mentales, tercera edición. Editorial Ars Médica, 2005. Disponible en: http://www. suicidioprevencion.com/pdf/RTM3.pdf [Consultado el 21 de julio de 2014]. 
13. Bertolote JM, Fleischmann A. Suicidal behavior prevention: WHO perspectives on research. American Journal of Medical Genetics Part C: Seminars in Medical Genetics 2005; 133 (1): 8-12.

14. Gómez-Durán EL, Forti-Buratti MA, Gutiérrez-López B, Belmonte-Ibáñez A, Martin-Fumadó C. Psychiatric disorders in cases of completed suicide in a hospital area in Spain between 2007 and 2010. Rev Psiquiatr Salud Ment 2014 Jul 1. pii: S1888-9891(14)00038-X. doi: 10.1016/j.rpsm.2014.02.001. [Epub ahead of print].

15. Hawton K, Saunders K, Topiwala A, Haw C. Psychiatric disorders in patients presenting to hospital following self-harm: a systematic review. J Affect Disord. 2013; 151 (3): 821-30.

16. Bolton JM, Walld R, Chateau D, Finlayson G, Sareen J. Risk of suicide and suicide attempts associated with physical disorders: a population-based, balancing score-matched analysis. Psychol Med 2014; 17: 1-10.

17. Pompili M, Forte A, Palermo M, Stefani H, Lamis DA, Serafini G. et al. Suicide risk in multiple sclerosis: a systematic review of current literature. J Psychosom Res 2012; 73 (6): 411-7.

18. Large M, Smith G, Sharma S, Nielssen O, Singh SP. Systematic review and meta-analysis of the clinical factors associated with the suicide of psychiatric in-patients. Acta Psychiatr Scand 2011; 124 (1): 18-29.

19. Large M, Sharma S, Cannon E, Ryan C, Nielssen O. Risk factors for suicide within a year of discharge from a psychiatric hospital: a systematic review. Aust NZ J Psychiatry 2011; 45: 619-28.

20. Ryan C, Nielssen O, Paton M, Large M. Clinical decisions in psychiatry should not be bassed on risk assessment. Australas Psychiatry 2010; 18 (5): 398-403.

21. Baader T, Richter P, Mund C. Suicidios de pacientes psiquiátricos hospitalizados y sus factores de riesgo. Un estudio caso-control. Rev Chil Neuro-Psiquiat 2004; 42 (4): 293-316.

22. King EA, Baldwin DS, Sinclair JM, Campbell MJ. The Wessex Recent In-Patient Suicide Study, 2. Case-control study of 59 in-patient suicides. Br J Psychiatry 2001; 178: 537-42.

23. Aedo A, Santander J, Nahuelpán E, Rodríguez J, Santander R. Caracterización del suicidio en centros de detención de la Región Metropolitana. Rev Chil Neuro-Psiquiat 2013; 51 (Supl 1): 52.

24. Rodríguez J, Santander J, Nahuelpán E, Aedo A, San- tander R. Caracterización de suicidios ocurridos en hospitales generales y psiquiátricos de la Región Metropolitana de Chile. Rev Chil Neuro-Psiquiat 2013; 51 (Supl 1): 90.

25. Jones RM, Hales H, Butwell M, Ferriter M, Taylor PJ. Suicide in high security hospital patients. Soc Psychiatry Psychiatr Epidemiol 2011; 46 (8): 723-31.

26. Ryan C, Large S. Suicide risk assessment: where are we now? MJA 2013; 198 (9): 462-3.

27. Ministerio de Salud, República de Chile, Departamento de Asesoría Jurídica. Reglamento para la internación de las personas con enfermedades mentales y sobre los establecimientos que la proporcionan. En: República de Chile, Ministerio de Salud. DTON570/98. D.OF. 14.07.00; 2000.

28. Santander J, Flores L, Huerta D, Weiss C, Fuentes X. Diferencias clínicas y sociodemográficas en la hospitalización con y sin consentimiento de pacientes psiquiátricos en Chile. Acta Psiquiatr Psicol Am Lat 2012; 58 (1): 22-8.

29. Santander J, Huerta D, Aceituno D, Fuentes X. Descripción clínica y socio-demográfica de pacientes internados involuntariamente bajo el régimen de internación administrativa en Chile. Rev Chil Neuro-Psiquiatr 2011; 49 (2): 128-35.

30. Vargas T. La imprudencia médica, Algunos problemas de imputación de lo injusto penal. RDUCN, Coquimbo 2010; 17 (2): 99-132.

31. Urruela A, Romeo S. Tendencias actuales de la jurisprudencia española en materia de responsabilidad penal médica. Revista de Derecho Penal 2011; 34: 35-74.

32. Escobar F. Responsabilidad médica del psiquíatra. Rev Colomb Anestesiol 2012; 40 (1): 17-20.

33. Ho AO. Suicide: rationality and responsibility for life. Can J Psychiatry 2014; 59 (3): 141-7.

34. Chemtob CM, Hamada RS, Bauer G, Kinney B, Torigoe RY. Patients' suicides: frequency and impact on psychiatrists. Am J Psychiatry 1988; 145 (2): 224-8.

35. Prabhakar D, Anzia JM, Balon R, Gabbard G, Gray E, Hatzis N, et al. "Collateral damages": preparing residents for coping with patient suicide. Acad Psychiatry 2013; 37 (6): 429-30.

36. Prabhakar D, Balon R, Anzia JM, Gabbard GO, Lomax JW, Bandstra BS, et al. Helping Psychiatry Residents Cope with Patient Suicide. Acad Psychiatry 2014 Mar 25. [Epub ahead of print]. 